\section{Risk of ovarian malignancy algorithm}

S. Holdenrieder

Institut für Laboratoriumsmedizin, Deutsches Herzzentrum München, Klinik an der Technischen Universität München, München, Deutschland

\section{Synonym(e) ROMA}

Definition Der ROMA-Index kombiniert die Aussagekraft der Marker $>$ Carbohydrate antigen 125 (CA 125) und „human epidydimis 4“ (HE4) zu einem $>$ Risikoindex für das Vorliegen eines Ovarialkarzinoms. Beide Marker haben eine zueinander additive Sensitivität $(\triangleright$ Sensitivität, diagnostische).

HE4 ist zudem seltener bei differenzialdiagnostisch relevanten benignen gynäkologischen Erkrankungen erhöht, was zu einer höheren Spezifität v. a. bei prämenopausalen Frauen beiträgt. Der ROMA berechnet sich für prä- und postmenopausale Frauen verschieden:
- Prämenopausal: PI $=-12,0+2,38 \times \ln$ (HE4) $+0,0626 \times \ln (\mathrm{CA} 125$.

- Postmenopausal: PI $=-8,09+1,04 \times \ln$ $($ HE4 $)+0,732 \times \ln ($ CA 125 .

Die Wahrscheinlichkeit für das Vorliegen eines Ovarialkarzinoms ist dann:

$$
\text { ROMA - Wert }(\%)=\exp (\mathrm{PI}) /[1+\exp (\mathrm{PI})] \times 100
$$

Hierbei werden je nach Testhersteller unterschiedliche Grenzwerte für die Risikostratifikation angegeben.

Diagnostische Wertigkeit Risikostratifizierung, Differenzialdiagnose.

\section{Literatur}

Moore RG et al (2008) Gynecol Oncol 108:402-408

Moore RG et al (2010) Am J Obstet Gynecol 203(228):e1-e6 